\title{
Abnormal Achromatic and Chromatic Contrast Sensitivity in Neurofibromatosis Type 1
}

\author{
Maria José Ribeiro, ${ }^{1}$ Inês Ribeiro Violante, ${ }^{1}$ Inês Bernardino, ${ }^{1}$ Fabiana Ramos, ${ }^{2}$ \\ Jorge Saraiva, ${ }^{2}$ Pablo Reviriego, ${ }^{3}$ Meena Upadbyaya, ${ }^{3}$ Eduardo Duarte Silva, ${ }^{1}$ \\ and Miguel Castelo-Branco ${ }^{1}$
}

Purpose. Neurofibromatosis type 1 (NF1) is a monogenic disorder with the majority of patients presenting subtle to moderate cognitive impairments. Visuospatial deficits are considered to be one of the hallmark characteristics of their cognitive profile. However, low-level visual processing has not been previously investigated. Our aim was to study contrast perception in these patients to assess the function of early visual areas.

Methods. Contrast sensitivity was tested in 19 children and adolescents with NF1 and 33 control children and adolescents and 12 adults with NF1 and 24 control adults. The tasks used probed two achromatic spatiotemporal frequency channels and chromatic red-green and blue-yellow pathways.

RESUlTs. Individuals with NF1 showed significant contrast sensitivity deficits for the achromatic higher spatial frequency channel $\left[F_{(1,83)}=36.1, P<0.001\right]$ and for the achromatic low spatial high temporal (magnocellular) frequency channel $\left[F_{(1,72)}=8.0, P<0.01\right]$. Furthermore, individuals with NF1 presented a significant deficit in chromatic red-green (parvocellular) contrast sensitivity $(P<0.01)$ but not in blue-yellow (koniocelular) sensitivity. The decrease in achromatic sensitivity for higher spatial frequency was observed throughout the visual field, in both central and peripheral locations. In contrast, central contrast sensitivity for the magnocellular-biased condition was relatively preserved and only peripheral sensitivity was affected. Interestingly, the same pattern of deficits was found in both age groups tested.

Conclusions. These findings showed that contrast sensitivity is impaired in patients with NF1, associating for the first time abnormal low-level vision to the cognitive profile of this disorder. (Invest Ophthalmol Vis Sci. 2012;53:287-293) DOI: 10.1167/iovs.11-8225

From the ${ }^{1}$ Visual Neuroscience Laboratory, Institute of Biomedical Research in Light and Image, Faculty of Medicine, University of Coimbra, Coimbra, Portugal; the ${ }^{2}$ Medical Genetic Department, Pediatric Hospital of Coimbra, Coimbra, Portugal; and the ${ }^{3}$ Institute of Medical Genetics, Cardiff University, Heath Park Campus, Cardiff, Wales, United Kingdom.

Supported in part by Institute of Interdisciplinary Research of the University of Coimbra Grant III/14/2008 and Portuguese Foundation for Science and Technology Grants PIC/IC/83155/2007, SFRH/BPD/ 34392/2006, SFRH/BD/41348/2007, and SFRH/BD/41401/2007.

Submitted for publication July 15, 2011; revised September 14 and November 14, 2011; accepted December 9, 2011

Disclosure: M.J. Ribeiro, None; I.R. Violante, None; I. Bernardino, None; F. Ramos, None; J. Saraiva, None; P. Reviriego, None; M. Upadhyaya, None; E.D. Silva, None; M. Castelo-Branco, None

Corresponding author: Maria José Ribeiro, Visual Neuroscience Laboratory, IBILI, Faculty of Medicine, University of Coimbra, Az. de Sta Comba, 3000-548 Coimbra, Portugal; mjribeiro@fmed.uc.pt.
$\mathrm{N}$ eurofibromatosis type 1 (NF1) is a monogenic disorder with an incidence of around 1:3500. ${ }^{1} \mathrm{~A}$ high percentage of patients with NF1 show cognitive deficits. ${ }^{2}$ In particular, visuospatial deficits are considered to be one of the hallmark characteristics of their cognitive profile. ${ }^{3,4}$ In addition, individuals with NF1 underperform in receptive and expressive language assessments, tests of executive function, global attention, motor speed, and coordination. ${ }^{3,5,6}$ Children with NF1 are also more likely to develop attention-deficit hyperactivity disorder, present in 30 to $50 \%$ of patients. ${ }^{3}$ The origin and extent of these problems is not fully understood. ${ }^{3}$ One possibility is that deficits in low-level vision are present and may underlie, at least in part, the NF1 cognitive problems. Indeed, one functional magnetic resonance imaging study revealed decreased activation of the occipital visual cortex of these patients. ${ }^{7}$ Yet, low-level vision has not been studied before in NF1.

In early stages of visual processing, information is transmitted from the retina to the visual cortex by three parallel physiologically defined pathways, that is, parvo-, magno-, and koniocellular. These neural pathways may underlie the detection mechanisms defined psychophysically. ${ }^{8,9}$ The parvocellular pathway is thought to underlie fine discrimination of visual features, particularly in the central visual field, and is sensitive to modulation of long-wavelength (L) and middle-wavelength (M) cone contrast, which results in red-green chromatic contrast. ${ }^{10}$ In addition, the parvocellular channel also responds to achromatic stimulation with high spatial and low temporal frequencies. ${ }^{11}$ On the other hand, the magnocellular pathway is most sensitive to achromatic stimuli with low spatial and high temporal frequencies. The magnocellular pathway is thought to play an important role in spatial localization and motion processing. ${ }^{12}$ The function of the koniocellular pathway is less understood. ${ }^{13}$ It most certainly plays a role in color vision due to its sensitivity to short-wavelength (S) cone (blueyellow) contrast. In addition, it might be involved in spatial processing, as suggested by its significant response to peripheral stimulation, ${ }^{14,15}$ and in motion perception. ${ }^{16,17}$ Thus, specific deficits in each of these independent pathways might have specific implications in the visual profile of these patients and, thus, it is important that we understand the origin of the visual impairments investigated.

With this in mind, we tested contrast sensitivity in children and adolescents with NF1 and adults with NF1 using low-level visual stimulation that preferentially activate the red-green (parvocellular), blue-yellow (koniocellular), or low spatial high temporal frequency (strongly magnocellular biased) channels. In addition, we also tested a parvocellular-biased achromatic channel (stimuli with higher spatial and lower temporal frequency). Furthermore, we studied if the dependence of contrast sensitivity on eccentricity was altered. Parvo- and magnocellular pathways differ in the way their responses de- 
pend on the eccentricity of the visual stimuli, with the magnocellular pathway dominating at more peripheral locations. ${ }^{18}$ Thus, impairments of these pathways in central or peripheral locations could have different behavioral consequences that should be considered.

\section{MeTHODS}

\section{Participants: Recruitment and Exclusion Criteria}

Thirty-five families with NF1 were recruited in collaboration with the Genetics Department of the Pediatric Hospital of Coimbra in Portugal All participants met the National Institutes of Health Consensus Development Conference clinical criteria for NF1. ${ }^{19}$ We excluded patients with known brain pathology or ophthalmological problems that could influence the results. Furthermore, to ensure that the patients included in the study had no unknown brain pathology (e.g., optic gliomas), they were submitted to magnetic resonance structural scans (MP-RAGE [magnetization-prepared rapid gradientecho] and FLAIR [fluid-attenuated inversion recovery] sequences). Standard neuroradiological assessments were carried out by an experienced neuroradiologist. Significant structural anomalies, besides T2-hyperintensities, were identified in four individuals with NF1. These were excluded from this study.

In addition, all patients with NF1 were submitted to a complete ophthalmic examination, including best-corrected visual acuity, stereopsis evaluation (Randot Stereotest; Stereo Optical Co., Chicago, IL), slit-lamp examination of anterior chamber structures, and fundus examination. Lisch nodules were observed in $55 \%$ of patients but no anomalies that could affect vision were found.

For the control group, we recruited unaffected siblings $(n=3)$ and parents and spouses of patients with NF1 $(n=7)$. Additional healthy children and adolescents were recruited from a school with wide socioeconomic coverage $(n=30)$, whereas additional healthy adults were recruited from an adult further education center. These typically developing participants had no history of learning, developmental, cognitive, neurologic, or neuropsychiatric problems.

Thus, for analysis, we included 19 children and adolescents with NF1 and 33 control children and adolescents. In the adult groups, we included 12 individuals with NF1 and 23 controls. There were no statistical differences for age and education level between the NF1 and control groups. Furthermore, there were no significant differences between ages of female participants of NF1 and control groups. The same was true for male participants. Furthermore, sex ratios were similar in both groups. Characteristics of participants are summarized in Table 1 .

Parents of children on stimulant medication (methylphenidate) were requested not to give the medication on the days of testing (three children with NF1), ensuring that these children were not under the influence of methylphenidate during testing.

\section{Protocol Approvals and Patient Consents}

The study was conducted in accordance with the tenets of the Declaration of Helsinki and was approved by the Ethics Committees of the Faculty of Medicine of Coimbra and of the Children's Hospital of Coimbra. Written informed consent was obtained from participants older than 17 years of age and from the legal representative in the case of participants younger than 18 years of age, after explanation of the nature and possible consequences of the study. Children and adolescents younger than 18 years of age gave written or oral informed consent.

\section{Genetic Characterization of Participants with NF1}

To further characterize our NF1 population, patients' DNA was extracted from peripheral blood using standard procedures, and whole gene sequencing and multiplex ligation-dependent probe amplification analysis were carried out with the aim of identifying the disease causing NF1 mutations ${ }^{20}$ (see Results section).

\section{Neuropsychological Characterization of Participants}

In all participants with NF1 younger than 17 years of age $(n=18)$ and in a subgroup of control children and adolescents $(n=18)$, we administered the Portuguese adapted version of the Wechsler Intelligence Scale for Children (WISC-III). ${ }^{21}$ For participants older than 17 years of age, we applied the 12-item short form of the Raven Advanced Progressive Matrices ${ }^{22}$ as an indication of nonverbal intelligence. Participants also performed Benton's Facial Recognition Test (FRT) ${ }^{23}$ and Benton's Judgment of Line Orientation (JLO) (Form H). ${ }^{24}$ Two adult control participants and one child with NF1 did not perform the FRT and JLO tests.

Adults performed all tests (contrast sensitivity, nonverbal intelligence, visuospatial, and visuoperceptual tests) on the same day with testing duration of approximately 1.75 hours, including breaks. Chil-

TABLE 1. Characteristics of NF1 and Control Groups

\begin{tabular}{|c|c|c|c|c|c|c|}
\hline \multirow[b]{2}{*}{ Factor } & \multicolumn{3}{|c|}{ Children and Adolescents } & \multicolumn{3}{|c|}{ Adults } \\
\hline & NF1 & Controls & $\boldsymbol{P}$ & NF1 & Controls & $\boldsymbol{P}$ \\
\hline Age, y & & & & & & \\
\hline $\begin{array}{l}\text { Mean } \pm \text { SD } \\
\text { Range }\end{array}$ & $\begin{array}{c}11.5 \pm 2.5 \\
7-17\end{array}$ & $\begin{array}{c}11.7 \pm 2.3 \\
7-16\end{array}$ & 0.74 & $\begin{array}{c}33.3 \pm 4.1 \\
26-38\end{array}$ & $\begin{array}{c}34.8 \pm 5.8 \\
26-45\end{array}$ & 0.43 \\
\hline Sex ratio & & & & & & \\
\hline $\begin{array}{l}\text { \% Female } \\
\text { Education level, y }\end{array}$ & $63 \%$ & $61 \%$ & 0.86 & $92 \%$ & $91 \%$ & 0.97 \\
\hline $\begin{array}{l}\text { Mean } \pm S D \\
\text { Range }\end{array}$ & - & - & & $\begin{array}{c}8.4 \pm 3.8 \\
4-16\end{array}$ & $\begin{array}{c}10.4 \pm 4.3 \\
4-17\end{array}$ & 0.19 \\
\hline $\begin{array}{l}\text { Full-scale IQ (WISC-III) } \\
\text { Mean } \pm \text { SD }\end{array}$ & $96 \pm 15$ & $111 \pm 15$ & $<0.01$ & - & - & \\
\hline $\begin{array}{l}\text { Raven score } \\
\quad \text { Mean } \pm \text { SD }\end{array}$ & - & - & & $6.7 \pm 2.7$ & $8.3 \pm 2.9$ & 0.12 \\
\hline $\begin{array}{l}\text { Benton's JLO } \\
\text { Mean } \pm \text { SD }\end{array}$ & $17.5 \pm 6.1$ & $24.0 \pm 4.2$ & $<0.01$ & $19.3 \pm 4.1$ & $22.8 \pm 5.7$ & 0.07 \\
\hline $\begin{array}{l}\text { Benton's FRT } \\
\text { Mean } \pm \text { SD }\end{array}$ & $20.1 \pm 2.1$ & $21.6 \pm 2.3$ & $<0.05$ & $22.3 \pm 1.8$ & $23.2 \pm 1.9$ & 0.21 \\
\hline
\end{tabular}

$P$ values were relative to $t$-test comparisons between NF1 and control groups, except for comparisons of sex ratio where chi-square tests were used, and comparisons of JLO scores where the Mann-Whitney test was used. 
dren and adolescents performed all tests except the WISC-III that was performed on a subsequent visit to the institute.

The neuropsychological characterization of the groups is summarized in Table 1. As expected, full-scale IQ of children and adolescents with NF1 was significantly lower than full-scale IQ of controls $(P<$ 0.01 ). For the adult groups, although the NF1 Raven scores showed a tendency to be lower than control levels, this difference was not significant. Furthermore, the performance on the JLO test was significantly impaired in the children and adolescent groups $(P<0.001)$. Also, in the adult groups, these scores were lower in the NF1 group, but the difference did not reach significance $(P=0.07)$. Interestingly, children and adolescents with NF1 were significantly impaired on the FRT $(P<0.05)$, suggesting impaired visuoperceptual abilities. However, we did not find a significant difference for the adults' performance on this test.

\section{Achromatic Contrast Sensitivity Test for Low Temporal and Higher Spatial Frequency Channel}

This contrast sensitivity test used static achromatic vertical gratings, with an intermediate spatiotemporal profile aimed at biasing the activation of the parvocellular pathway (for more specific modulation of this pathway, see the following text on chromatic tests). This task was based on a custom-made task described before. ${ }^{18}$ Stimuli were static vertical gratings, with a spatial frequency of 3.5 cycles per degree (mean background luminance of $51 \mathrm{~cd} / \mathrm{m}^{2}$ ), displayed on a 21-inch monitor (Trinitron GDM-F520 Sony monitor). The stimulus sizes, shapes, and locations are represented in Figure 1A. The width of each stimulus was $10^{\circ}$ of visual angle ( 35 grating cycles). Stimulus duration was $200 \mathrm{~ms}$ and the interstimulus interval varied randomly between 2300 and $2800 \mathrm{~ms}$. The stimuli were presented with no temporal filter pseudorandomly within nine locations of the visual field (Fig. 1A). Participants were positioned comfortably at a viewing distance of 36 $\mathrm{cm}$ and were instructed to fixate the black square in the center of the screen and report the presence of "striped" targets (detection task) in any of the nine locations by means of a button press. A chin and forehead rest was used to ensure a stable viewing position throughout testing. Participants' reliability was evaluated by the inclusion of falsepositive ( $0 \%$ contrast stimuli) and false-negative ( $100 \%$ contrast) "catch trials." Trials with false positives or false negatives $\geq 33 \%$ were aborted or, if run until the end, excluded from analysis, according to standard criteria. The trial was then repeated after a small rest period. If in repeated trials the participant still responded with a high number of false positives or false negatives, then all data from that participant were not used in analysis.

During task performance the experimenter visually monitored each participant's fixation. If the participant was not able to maintain fixation the test was aborted and a second trial was run after repetition of test instructions.

This task was performed in a monocular way for both eyes, with the first tested eye being chosen in a random manner and an opaque patch occluding the other eye.

Luminance contrast of the stimulus was expressed according to Michelson. Contrast sensitivity results were expressed in terms of decibel $(\mathrm{dB})$ units, $\mathrm{dB}=20 \times \log (1 / c)$, with contrast $c$ measured as a percentage. To obtain the psychophysical thresholds, the test uses nine randomly interleaved logarithmic staircases, one for each location tested. The contrast value used for a given trial was calculated using the previous trial value plus or minus the step size in $\mathrm{dB}$. The initial step size used was $3 \mathrm{~dB}$. Staircases were run for a total of four reversals, with the contrast at the final two reversals being averaged to estimate the contrast threshold.

For analysis, we averaged the contrast sensitivity values of each visual field location of both eyes for each individual. Furthermore, to study the effect of eccentricity, we calculated the average contrast sensitivity of three zones: $0-5$, the central location with eccentricity between 0 and $5^{\circ}$ of visual angle (light gray circular region in Fig. 1A); $5-10$, averaged contrast sensitivity levels of the four locations with
A

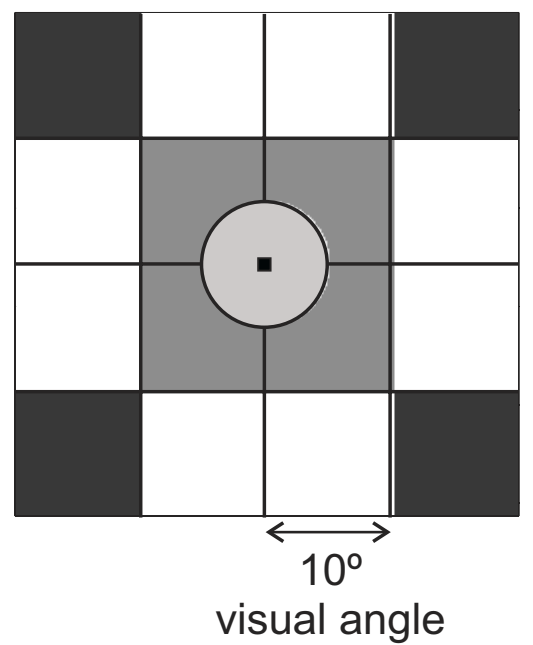

B

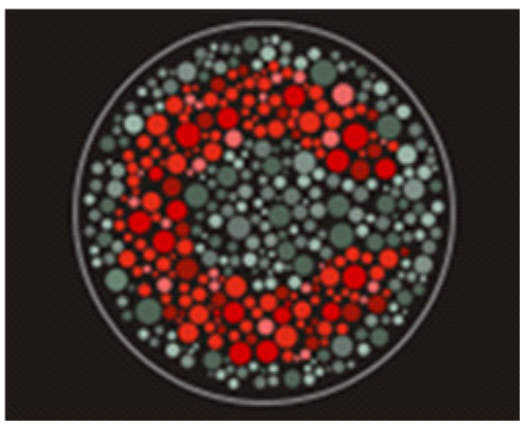

C

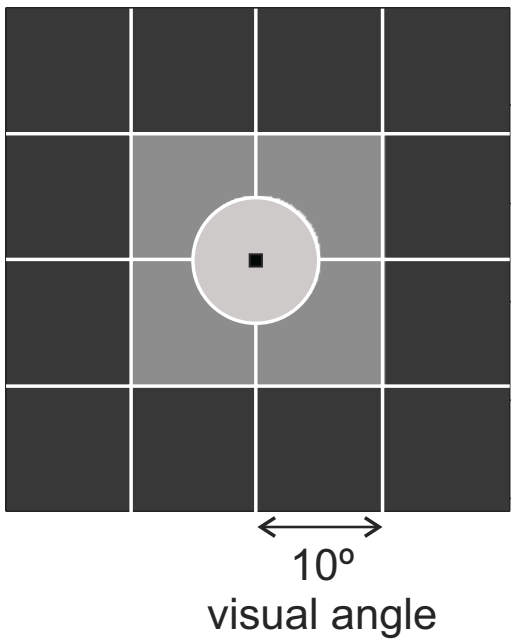

FigURE 1. Schematic representation of the contrast sensitivity tests used. (A) Schematic representation of the sizes and shapes of the nine locations within the visual field where the stimuli used in the achromatic contrast sensitivity test for low temporal and higher spatial frequency channel were presented. The black square in the middle of the figure represents the fixation square. (B) Schematic illustration of the stimuli used in the chromatic sensitivity tests (Cambridge Color Test) representing a luminance noise stimulus with superimposed chromatic target (Landolt C shape, colored in red). (C) Schematic representation of the sizes and shapes of 17 locations within the visual field where the stimuli used in the achromatic contrast sensitivity test for high temporal and low spatial frequency channel (magnocellular) were presented. The two nasal locations are not shown. The black square in the middle of the figure represents the fixation square. 
eccentricity between 5 and $10^{\circ}$ (four gray regions adjacent to circular region in Fig. 1A); and 10-20, averaged contrast sensitivity levels of the four outermost locations (eccentricity between 10 and $20^{\circ}$; four darker gray squares in Fig. 1A).

\section{Chromatic Sensitivity Tests}

We probed chromatic contrast sensitivities using a standard commercial color vision test (Cambridge Color Test [CCT]; Cambridge Research Systems, Rochester, UK), a computer-controlled psychophysical method. ${ }^{25}$ Stimuli were displayed on a 21 -inch monitor (GDM-F520; Sony, Tokyo, Japan). Participants viewed a static pattern of circles of various sizes and luminances with superimposed chromatic contrast defining a C (gap size: $1.6^{\circ}$; outer diameter: $7.6^{\circ}$; inner diameter: $3.81^{\circ}$; viewing distance: $1.8 \mathrm{~m}$; Fig. 1B) and were instructed to indicate whether the gap was facing up, down, left, or right, by pressing one of four buttons. A chin and forehead rest was used to ensure a stable viewing position throughout testing. We used a color version of the test (Trivector; CCT), where the targets differ from the background along one of the three color confusion lines: protan, deutan, and tritan. The test uses three randomly interleaved staircases to dynamically adjust the chromaticity of the target according to the participant's performance to establish the chromaticity difference between target and background needed for reliable report of the orientation of the $\mathrm{C}$. Occasional control trials, with a target presented at maximal chromatic saturation, were introduced to ensure that the participant was alert. Testing on any one staircase was terminated after 11 reversals and the mean of the last 6 reversals was taken as the threshold estimate for the direction being tested.

The test was performed in a monocular way, with the eye tested first chosen in a random manner and an opaque patch occluding the other eye. An initial session of training ensured all participants understood the task.

The chromatic contrast along each of three color confusion lines, protan, deutan, and tritan, activates only one type of cone photoreceptors. Colors within the protan line activate the L-cone type, within deutan activate the M-cone type, and within the tritan line activate the S-cone type. We took as the threshold for the red-green (parvocellular) chromatic channel the average of the thresholds along protan and deutan lines and the threshold along the tritan line as the blue-yellow (koniocellular) contrast threshold. Note that high chromatic thresholds relate to low-contrast sensitivity.

\section{Achromatic Contrast Sensitivity Test for High Temporal and Low Spatial Frequency Channel (Magnocellular)}

Magnocellular contrast sensitivity was measured using a commercially available system (Humphrey Matrix perimeter, Welch Allyn, Skaneateles, NY; Zeiss Humphrey Systems, Dublin, CA). Stimuli were sinusoidal vertical gratings with high temporal frequency $(25 \mathrm{~Hz})$ and low spatial frequency ( 0.25 cycles per degree) frequency-doubling stimuli. We used a strategy (N-30-F) that tests a total of 19 locations (four $10^{\circ}$ square targets per quadrant and a central $10^{\circ}$ diameter circular target plus two nasal locations; Fig. 1C). During testing, stimuli were presented successively with each of the 19 target locations selected on a random basis. Each stimulus was presented for a maximum of $720 \mathrm{~ms}$. During the first $160 \mathrm{~ms}$, stimulus contrast was increased gradually from zero to the contrast selected for that trial. If the stimulus was not seen, it remained at that contrast for up to $400 \mathrm{~ms}$ and was then gradually decreased to zero during the final $160 \mathrm{~ms}$. Between stimulus presentations, there was an interval of up to $500 \mathrm{~ms}$, with duration randomly defined. This test uses a staircase threshold strategy known as Modified Binary Search, with a four-reversals rule for determining the threshold level and a dynamic luminance ratio range from 56 to $0 \mathrm{~dB}$.

The instrument has a sliding visor that automatically occludes one of the eyes, allowing monocular viewing. The first eye tested was chosen in a random manner. Participants were instructed to rest their forehead on the visor and fixate the black square in the center of the screen and report the presence of "striped" targets. Furthermore, a brief practice trial ensured that the participant understood the task. During testing, the experimenter visually monitored fixation, by attending the image of the participant's eye displayed on the experimenter screen. The test was aborted if the participant was not able to maintain fixation. Furthermore, if necessary, the test was paused to allow the participant to rest. In addition, performance reliability was assessed by monitoring fixation loss using stimuli placed on the blind spot and by computing false positive and negative errors. Tests with false positives, false negatives, or fixation losses $\geq 33 \%$ were aborted or, if run until the end, excluded from analysis, according to standard criteria. The trial was then repeated after a small rest period. If in repeated trials the participant still responded with a high number of errors, then all data from that participant were not used in the analysis.

This frequency-doubling perimetry has been shown to reveal a significant difference between the first eye tested compared with the second eye tested. ${ }^{26}$ Therefore, we analyzed our data comparing the first eye with the second eye tested. This eye order effect is due to monocular light adaptation and appears when the second eye is tested shortly after the first one. In participants where testing of one of the eyes had to be repeated (due to a high number of false positives, false negatives, or fixation errors) this sequence was disrupted, thereby changing the eyes' state of adaptation. This difference in timings between eye tests might interfere with contrast sensitivity levels. Thus, we chose to analyze data only from the participants that performed successfully the tests of one eye after the other without repetitions. After excluding these participants, we remained with 16 children and adolescents with NF1, 30 control children and adolescents, and 10 adults with NF1 and 20 control adults. As expected, we found a significant order effect of the eye tested $\left[F_{(1,72)}=15.9, P<0.001\right]$, with the average contrast sensitivity of the second eye significantly lower than the sensitivity of the first eye. No significant interaction was found between eye and clinical group or between eye and age group. These findings indicated that the effect of eye order testing was the same for controls and participants with NF1. Thus, for each participant, we averaged the contrast sensitivity of both eyes, for each visual field location.

To study the effect of eccentricity, we averaged the levels of contrast sensitivity in three zones: 0 to 5 (the central location with eccentricity between 0 and $5^{\circ}$ of visual angle; light gray circular region in Fig. 1C); 5 to 10 (4 locations with eccentricity between 5 and $10^{\circ}$; 4 gray regions adjacent to circular region in Fig. 1C); and 10 to 20 (12 locations with eccentricity between 10 and $20^{\circ}$; darker gray squares in Fig. 1C).

\section{Statistical Analysis}

All statistical analyses were performed with a commercial statistics/ analytical program (PAWS Statistics 18; SPSS Inc., Chicago, IL). We verified the normality assumption for the different parameters using the Shapiro-Wilk test. All measures were normally distributed except the chromatic thresholds and the scores from the JLO test. For the normally distributed data, we used repeated-measures ANOVA analyses, parametric $t$-tests, and Pearson's correlation analyses. For the nonnormally distributed data, we used the Mann-Whitney test and Spearman's correlation analyses.

\section{Results}

\section{Genetic Characterization of the Individuals with NF1}

In our cohort of patients with NF1, we identified 23 different NF1 mutations in 24 families. NF1 mutations were therefore identified in $69 \%$ of the families, confirming the NF1 diagnosis of the majority of the recruited patients with NF1. This percentage of mutation identification is in line with previous studies $^{27}$ (Table $2^{28-34}$ ). One of the nonsense mutations was found in two unrelated families. Two missense mutations re- 
TABLE 2. Summary of the Disease Causing NF1 Mutations Identified in 24 Families with NF1

Participating in This Study

\begin{tabular}{|c|c|c|c|}
\hline Mutation Type & $\begin{array}{c}\text { Mutation at Coding DNA } \\
\text { Level }\end{array}$ & $\begin{array}{c}\text { Mutation at Protein } \\
\text { Level }\end{array}$ & Reference \\
\hline \multirow[t]{7}{*}{ Nonsense } & c. $2041 \mathrm{C}>\mathrm{T}$ & p.Arg681X & Ars et al. ${ }^{28}$ \\
\hline & c. $2446 C>T$ & p.Arg816X & Maynard et al. ${ }^{29}$ \\
\hline & c. $3318 \mathrm{C}>\mathrm{G}$ & p.Tyr1109X & Not previously reported \\
\hline & c. $3942 \mathrm{G}>\mathrm{A}$ & p.Trp1314X & Upadhyaya et al..$^{30}$ \\
\hline & c. $4537 \mathrm{C}>\mathrm{T}$ & p.Arg1515X & Not previously reported \\
\hline & c. $5458 \mathrm{C}>\mathrm{T}$ & p.Gln1828X & Not previously reported \\
\hline & c. $574 \mathrm{C}>\mathrm{T}$ & p.Arg192X & Toliat et al. ${ }^{31}$ \\
\hline \multirow[t]{5}{*}{ Frameshift } & c.5565_5566 CTdel & p.Asn1963fsX8 & Not previously reported \\
\hline & C7206CAdel & p.His $2402 \mathrm{G} \operatorname{lnfX} 3$ & Not previously reported \\
\hline & c. $1329 \mathrm{del} \mathrm{T}$ & & Not previously reported \\
\hline & $\begin{array}{l}\text { c.del4815_4827 del } \\
\text { TGTCTTACTGACT }\end{array}$ & p.His $1605 \mathrm{X}$ & Not previously reported \\
\hline & c. $2500-2501$ ins C & & Not previously reported \\
\hline \multirow[t]{5}{*}{ Missense } & c. $2084 \mathrm{~T}>\mathrm{C}$ & p.Leu695Pro & Fahsold et al. ${ }^{32}$ \\
\hline & c. $2786 \mathrm{~T}>\mathrm{C}$ & p.Leu929Pro & Not previously reported \\
\hline & c. $3827 \mathrm{G}>\mathrm{C}$ & p.Arg1276Gln & Fahsold et al. ${ }^{32}$ \\
\hline & c. $5587 \mathrm{~T}>\mathrm{C}$ & p.Phe1863Leu & Not previously reported \\
\hline & c. $1466 \mathrm{~A}>\mathrm{G}$ & p.Tyr489Cys & Osborn and Upadhyaya 33 \\
\hline \multirow{5}{*}{ Splice site } & c. $730+1 G>A$ & IVS5 & Not previously reported \\
\hline & c. $1720+3 A>G$ & IVS 11 & Purandare et al. ${ }^{34}$ \\
\hline & c. $3196-2 \mathrm{~A}>\mathrm{C}$ & IVS $19 a$ & Not previously reported \\
\hline & c. $5944-6 A>G$ & IVS31 & Not previously reported \\
\hline & c. $8097+1 \mathrm{G}>\mathrm{A}$ & IVS 47 & Not previously reported \\
\hline Gross deletion & Exons $4 \mathrm{~A}, 4 \mathrm{~B}$ & & Not previously reported \\
\hline
\end{tabular}

ported in this study represent novel changes within the gene. None of these sequence changes has been identified in $>1000$ normal chromosomes studied for entire NF1 gene mutations. All these sequence changes are evolutionarily conserved in different species.

\section{Achromatic Contrast Sensitivity at an Intermediate Spatiotemporal Frequency Channel}

We measured the achromatic contrast sensitivity (with a parvocellular bias) for nine visual field locations in individuals with NF1 and control participants. Using repeated-measures analyses, we studied the effect of eccentricity, clinical group, and age group. We found a highly significant effect of clinical group $\left[F_{(1,83)}=36.1, P<0.001\right.$; Fig. 2] with no effect of age group (children/adolescents and adults) or interaction between clinical group and age group, indicating that the contrast sensitivity deficit was present in both children and adolescents with NF1 and adults with NF1. Furthermore, as expected, we found a significant effect of eccentricity $\left[F_{(1.7,137)}=444, P<0.001 ;\right.$ Fig. 2$]$ and a significant interaction between eccentricity and clinical group $\left[F_{(1.7,137)}=4.8, P<\right.$ 0.05], with the control group showing a greater decline of contrast sensitivity with eccentricity than that of the NF1 group (Fig. 2). In addition, we also found a significant interaction between eccentricity and age group $\left[F_{(1.7,137)}=9.6, P<\right.$ $0.001]$, with the adult participants showing a higher effect of eccentricity than that of the children and adolescents group, but no interaction between eccentricity, clinical group, and age group. Although there was an interaction between eccentricity and clinical group, the contrast sensitivity values of individuals with NF1 were always significantly lower than control levels for all eccentricities tested (post hoc $t$-tests: $P<$ 0.001; Fig. 2).

\section{Chromatic Contrast Sensitivity}

Interestingly, we found that, as for the achromatic parvocellular sensitivity, chromatic parvocellular thresholds were significantly affected in individuals with NF1 (thresholds in CIE
[International Commission on Illumination] $1976 L^{*}, u^{*}, v^{*}$ color space units, median [interquartile range, Q1-Q3]: control $=0.0051 \quad[0.0042-0.0062] ; \mathrm{NF} 1=0.0061 \quad[0.0055-$ $0.0070]$; nonparametric test, $P<0.01)$. This difference was significant both for children and adolescents $(P<0.05)$ and for adults $(P<0.05)$, separately. In contrast, the koniocellular thresholds were not significantly different between the groups (median [interquartile range, Q1-Q3]: control $=0.0075$ [0.0060-0.0098]; NF1 $=0.0084$ [0.0071-0.0100]; n.s.).

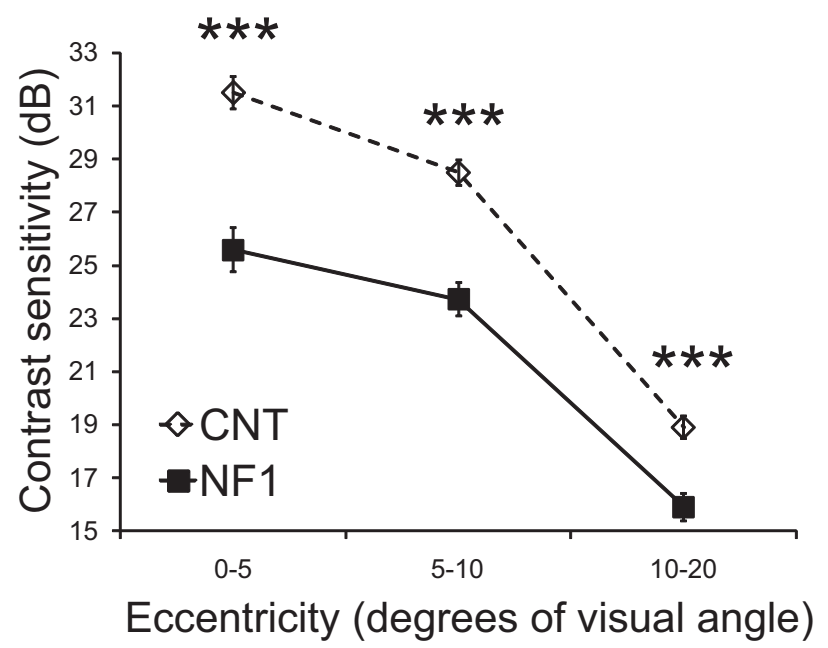

FIGURE 2. Individuals with NF1 showed decreased contrast sensitivity at an intermediate spatiotemporal frequency channel (parvocellularbiased). The graph shows contrast sensitivity versus eccentricity in both control participants and individuals with NF1. Contrast sensitivities at eccentricities $0-5^{\circ}, 5-10^{\circ}$, and $10-20^{\circ}$ were calculated as the average of the sensitivities of the central locations of both eyes, of the four locations with eccentricity between 5 and $10^{\circ}$ of both eyes and of the 4 locations with eccentricity between 10 and $20^{\circ}$ of both eyes, respectively. All data are expressed as mean \pm SE. ${ }^{* * *} P<0.001$. 


\section{Magnocellular-Biased Contrast Sensitivity}

In addition, we studied the effect of eccentricity, clinical group, and age group in magnocellular-biased contrast sensitivity. Repeated-measures analysis revealed a significant effect of clinical group $\left[F_{(1,72)}=8.0, P<0.01\right.$; Fig. 3], no effect of age group, and no interaction between clinical group and age group. These results indicated a magnocellular-biased contrast sensitivity deficit present in both children and adolescents with NF1 and adults with NF1. Furthermore, we found a significant effect of eccentricity $\left[F_{(1.8,144)}=43.5, P<0.001\right.$; Fig. 3], a significant interaction between eccentricity and clinical group $\left[F_{(1.8,144)}=3.7, P<0.05\right]$ and no interaction between eccentricity and age group or between eccentricity, age group, and clinical group. The interaction between eccentricity and clinical group was associated with a steeper decline in contrast sensitivity with eccentricity in the NF1 group than that of the control group (Fig. 3). In fact, post hoc $t$-tests showed that the contrast sensitivity in the central location of the visual field was not significantly different between the clinical groups and only the more peripheral locations, from $5^{\circ}$ eccentricity onward, presented significant contrast sensitivity deficits $(P<0.05)$.

\section{Relation between NF1 Low-Level Visual Deficits and Cognitive Abilities}

NF1 contrast sensitivity values did not correlate with the individual neuropsychological data, that is, IQ (measured as fullscale IQ, for children and adolescents, and Raven scores, for adults), visuospatial skills (measured using the Benton's JLO test), and visuoperceptual skills (measured using the Benton's FRT). This lack of correlation suggests that the contrast sensitivity impairments found in NF1 are not explained by low intellectual abilities or deficits in visuospatial or visuoperceptual skills.

\section{Discussion}

In this study, we described, for the first time, the presence of low-level visual contrast sensitivity deficits in individuals with NF1 in the absence of signs of structural brain damage.

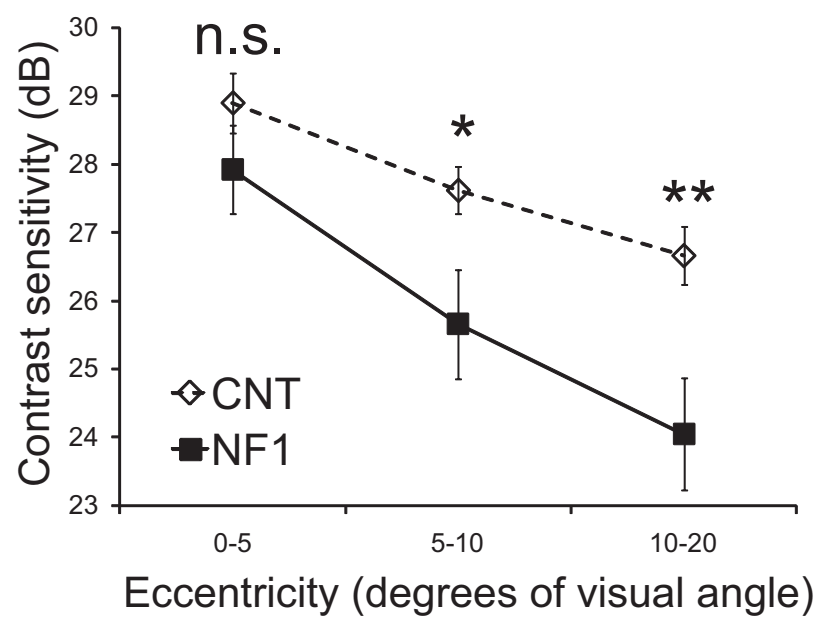

Figure 3. Magnocellular-biased contrast sensitivity is affected in individuals with NF1. The graph shows contrast sensitivity versus eccentricity in both controls and individuals with NF1. Contrast sensitivity at eccentricities $0-5^{\circ}, 5-10^{\circ}$, and $10-20^{\circ}$ were calculated, respectively, as the average of the sensitivities of the central locations of both eyes, of the four locations with eccentricity between 5 and $10^{\circ}$ of both eyes and of the 12 locations with eccentricity between 10 and $20^{\circ}$ of both eyes. All data are expressed as mean $\pm \mathrm{SE}$. ${ }^{*} P<0.05 ;{ }^{* *} P<0.01$.
The contrast sensitivity tasks used were chosen to bias activation of each of the three retinocortical pathways (parvo-, konio-, and magnocellular), with their different physiologic properties, although not completely isolating the function of each pathway. ${ }^{10}$ The intermediate spatial/low temporal frequency test and the chromatic red-green test activate mostly the parvocellular pathway. The low spatial/high temporal frequency test activates mostly the magnocellular pathway and the chromatic blue-yellow test activates mostly the koniocellular channel. Thus, our results suggest that individuals with NF1 present a pronounced parvocellular central and peripheral sensitivity deficit, impaired peripheral magnocellular sensitivity, and relatively spared central magno- and koniocellular function. The peripheral pattern of magnocellular impairment has relevant functional implications, given the dominance of this channel in peripheral vision and in terms of input to the dorsal stream, more specialized in motion detection and object localization. ${ }^{12}$

One of the main hypotheses explaining the pathophysiologic mechanism underlying the cognitive deficits observed in this disease indicates enhanced inhibitory neurotransmission. However, this hypothesis was tested only in NF1 mice models. ${ }^{35-37}$ Interestingly, our study, in humans, identified a pattern of visual impairments, deficient parvocellular contrast sensitivity, and relatively spared central magnocellular sensitivity, also observed in studies investigating the effect of benzodiazepines, drugs that enhance GABAergic (inhibitory) neurotransmission, in humans. ${ }^{38,39}$ Thus, our results are in line with the impaired inhibition hypothesis and might be of importance, given that they provide evidence that an indirect visual marker of inhibition (contrast sensitivity) is altered in this condition. The understanding of the nature of the neurophysiologic and psychophysical anomalies present in NF1 is important for the development of pharmacologic therapies targeting the cognitive impairments of these patients. Therefore, we believe that our study provides important evidence that helps predict the effects of drug therapies in these patients. We should note, nevertheless, that alternative causes for this contrast sensitivity deficit are possible. We do believe that future studies should directly address this question.

Contrast sensitivity has been shown to increase during childhood and adolescence, reaching adult levels in the late teens. ${ }^{40,41}$ However, in our study, we did not find an effect of age group. We believe that the lack of an age group effect might be because, although contrast sensitivity increases with age for children and adolescents, it declines with age for adults. ${ }^{41,42}$ This inverted $U$ shape of contrast sensitivity would result in no difference between the contrast sensitivity of group averages. Interestingly, the lack of an age effect in our data also suggests that the NF1 deficits in contrast sensitivity found in children and adolescents persist into adulthood.

Low-level visual deficits could underlie, at least in part, the NF1 cognitive impairments. However, we observed no correlation between contrast sensitivity, intelligence, visuospatial, and visuoperceptual abilities, suggesting that these deficits have independent causes. This observation is in line with our previous results in another neurodevelopmental model, Williams Syndrome. ${ }^{43}$ Individuals with NF1, like individuals with Williams Syndrome, are impaired in visuospatial processing, ${ }^{3,44}$ suggesting a dorsal stream deficit that could be the consequence of impaired magnocellular input. However, in line with our observation in Williams Syndrome, ${ }^{43}$ although we found a significant decrease in magnocellular sensitivity, these sensitivity levels did not correlate with visuospatial abilities, measured with the JLO test, or visuoperceptual abilities, measured with the Benton's FRT. Furthermore, the more severely affected parvocellular contrast sensitivity did not correlate with any of these neuropsychological tests, indicating that these low-level 
visual deficits are not the direct cause of these higher-order impairments.

In conclusion, we observed contrast sensitivity deficits in individuals with NF1, thereby identifying for the first time low-level visual impairments in this disorder. Further studies are needed to identify cognitive domains where deficits could be explained by decreased contrast sensitivity.

\section{References}

1. Tonsgard JH. Clinical manifestations and management of neurofibromatosis type 1. Semin Pediatr Neurol. 2006;13:2-7.

2. Hyman SL, Shores A, North KN. The nature and frequency of cognitive deficits in children with neurofibromatosis type 1. Neurology. 2005;65:1037-1044.

3. Levine TM, Materek A, Abel J, O'Donnell M, Cutting LE. Cognitive profile of neurofibromatosis type 1. Semin Pediatr Neurol. 2006 $13: 8-20$

4. Schrimsher GW, Billingsley RL, Slopis JM, Moore BD 3rd. Visualspatial performance deficits in children with neurofibromatosis type-1. Am J Med Genet A. 2003;120A:326-330.

5. Payne JM, Hyman SL, Shores EA, North KN. Assessment of executive function and attention in children with neurofibromatosis type 1: relationships between cognitive measures and real-world behavior. Child Neuropsychol. 2011;17:313-329.

6. Huijbregts S, Swaab H, de Sonneville L. Cognitive and motor control in neurofibromatosis type I: influence of maturation and hyperactivity-inattention. Dev Neuropsychol. 2010;35:737-751.

7. Clements-Stephens AM, Rimrodt SL, Gaur P, Cutting LE. Visuospatial processing in children with neurofibromatosis type 1. Neuropsychologia. 2008;46:690-697.

8. Kulikowski JJ, Tolhurst DJ. Psychophysical evidence for sustained and transient detectors in human vision. J Physiol. 1973;232:149162.

9. Krauskopf J, Williams DR, Heeley DW. Cardinal directions of color space. Vision Res. 1982;22:1123-1131.

10. Callaway EM. Structure and function of parallel pathways in the primate early visual system. J Physiol. 2005;566:13-19.

11. Merigan WH. Chromatic and achromatic vision of macaques: role of the P pathway. J Neurosci. 1989;9:776-783.

12. Merigan WH, Maunsell JH. How parallel are the primate visual pathways? Annu Rev Neurosci. 1993;16:369-402.

13. Hendry SH, Reid RC. The koniocellular pathway in primate vision. Annu Rev Neurosci. 2000;23:127-153.

14. Mullen KT, Dumoulin SO, McMahon KL, de Zubicaray GI, Hess RF. Selectivity of human retinotopic visual cortex to S-cone-opponent, L/M-cone-opponent and achromatic stimulation. Eur J Neurosci. 2007;25:491-502.

15. Vanni S, Henriksson L, Viikari M, James AC. Retinotopic distribution of chromatic responses in human primary visual cortex. Eur J Neurosci. 2006;24:1821-1831.

16. Sincich LC, Park KF, Wohlgemuth MJ, Horton JC. Bypassing V1: a direct geniculate input to area MT. Nat Neurosci. 2004;7:11231128.

17. Wandell BA, Poirson AB, Newsome WT, et al. Color signals in human motion-selective cortex. Neuron. 1999;24:901-909.

18. Silva MF, Maia-Lopes S, Mateus C, et al. Retinal and cortical patterns of spatial anisotropy in contrast sensitivity tasks. Vision Res 2008;48:127-135.

19. Neurofibromatosis. Conference statement. National Institutes of Health Consensus Development Conference. Arch Neurol. 1988; 45:575-578.

20. Upadhyaya M, Spurlock G, Kluwe L, et al. The spectrum of somatic and germline NF1 mutations in NF1 patients with spinal neurofibromas. Neurogenetics. 2009;10:251-263.

21. Menezes Rocha AM. Manual da Escala de Inteligência de Wechsler para crianças, de David Wechsler, adaptado e aferido para a população portuguesa por António Menezes Rocha. Lisbon: CEGOC; 2003.
22. Raven JC, Raven J, Court, JH. Manual for Raven's Progressive Matrices and Vocabulary Scales. Oxford, UK: Oxford Psychologists Press; 1993.

23. Benton AL, Hamsher KdeS, Varney NR, Spreen O. Contributions to Neuropsychological Assessment: A Clinical Manual. Oxford, UK: Oxford University Press; 1983.

24. Benton AL, Varney NR, Hamsher KD. Visuospatial judgment. A clinical test. Arch Neurol. 1978;35:364-367.

25. Regan BC, Reffin JP, Mollon JD. Luminance noise and the rapid determination of discrimination ellipses in colour deficiency. Vision Res. 1994;34:1279-1299.

26. Anderson AJ, McKendrick AM. Quantifying adaptation and fatigue effects in frequency doubling perimetry. Invest Ophthalmol Vis Sci. 2007; 48:943-948.

27. Griffiths S, Thompson P, Frayling I, Upadhyaya M. Molecular diagnosis of neurofibromatosis type 1: 2 years experience. Fam Cancer. 2007;6:21-34.

28. Ars E, Serra E, Garcia J, et al. Mutations affecting mRNA splicing are the most common molecular defects in patients with neurofibromatosis type 1. Hum Mol Genet. 2000;9:237-247.

29. Maynard J, Krawczak M, Upadhyaya M. Characterization and significance of nine novel mutations in exon 16 of the neurofibromatosis type 1 (NF1) gene. Hum Genet. 1997;99:674-676.

30. Upadhyaya M, Osborn MJ, Maynard J, Kim MR, Tamanoi F, Cooper DN. Mutational and functional analysis of the neurofibromatosis type 1 (NF1) gene. Hum Genet. 1997;99:88-92.

31. Toliat MR, Erdogan F, Gewies A, et al. Analysis of the NF1 gene by temperature gradient gel electrophoresis reveals a high incidence of mutations in exon 4b. Electrophoresis. 2000;21:541-544.

32. Fahsold R, Hoffmeyer S, Mischung C, et al. Minor lesion mutational spectrum of the entire NF1 gene does not explain its high mutability but points to a functional domain upstream of the GAPrelated domain. Am J Hum Genet. 2000;66:790-818.

33. Osborn MJ, Upadhyaya M. Evaluation of the protein truncation test and mutation detection in the NF1 gene: mutational analysis of 15 known and 40 unknown mutations. Hum Genet. 1999;105:327332.

34. Purandare SM, Lanyon WG, Connor JM. Characterisation of inherited and sporadic mutations in neurofibromatosis type-1. Hum Mol Genet. 1994;3:1109-1115.

35. Costa RM, Federov NB, Kogan JH, et al. Mechanism for the learning deficits in a mouse model of neurofibromatosis type 1. Nature. 2002; 415:526-530.

36. Cui Y, Costa RM, Murphy GG, et al. Neurofibromin regulation of ERK signaling modulates GABA release and learning. Cell. 2008; $135: 549-560$

37. Shilyansky C, Karlsgodt KH, Cummings DM, et al. Neurofibromin regulates corticostriatal inhibitory networks during working memory performance. Proc Natl Acad Sci USA. 2010;107:1314113146

38. Giersch A, Speeg-Schatz C, Tondre M, Gottenkiene S. Impairment of contrast sensitivity in long-term lorazepam users. Psychopharmacology (Berl). 2006;186:594-600.

39. Blin O, Mestre D, Paut O, Vercher JL, Audebert C. GABA-ergic control of visual perception in healthy volunteers: effects of midazolam, a benzodiazepine, on spatio-temporal contrast sensitivity. Br J Clin Pharmacol. 1993;36:117-124.

40. Beazley LD, Illingworth DJ, Jahn A, Greer DV. Contrast sensitivity in children and adults. Br J Ophthalmol. 1980;64:863-866.

41. Knoblauch K, Vital-Durand F, Barbur JL. Variation of chromatic sensitivity across the life span. Vision Res. 2001;41:23-36.

42. Adams CW, Bullimore MA, Wall M, Fingeret M, Johnson CA. Normal aging effects for frequency doubling technology perimetry. Optom Vis Sci. 1999;76:582-587.

43. Castelo-Branco M, Mendes M, Sebastiao AR, et al. Visual phenotype in Williams-Beuren syndrome challenges magnocellular theories explaining human neurodevelopmental visual cortical disorders. J Clin Invest. 2007;117:3720-3729.

44. Bellugi U, Lichtenberger L, Jones W, Lai Z, St George MI. The neurocognitive profile of Williams Syndrome: a complex pattern of strengths and weaknesses. J Cogn Neurosci. 2000;12(suppl 1): 7-29. 